\title{
Development of Computed Tomography Scanner Application: Prototype Model Approach
}

\author{
M.S.M. Yusoff ${ }^{1}$, R. Sulaiman ${ }^{1}$, K. Shafinah ${ }^{2,3}$, R. Fatihah ${ }^{2,4}$, J. Abdullah ${ }^{5}$ \\ ${ }^{1}$ Institute of Visual Informatics, National University of Malaysia (UKM), 43600 Bangi, Selangor, Malaysia. \\ ${ }^{2}$ Center for Artificial Intelligence Technology, Faculty of Information Science and Technology, National University of \\ Malaysia (UKM), 43600 Bangi, Selangor, Malaysia. \\ ${ }^{3}$ Faculty of Agriculture and Food Sciences, Universiti Putra Malaysia Bintulu Campus (UPMKB), Nyabau Road, P.O. \\ Box 396, 97008 Bintulu, Sarawak, Malaysia. \\ ${ }^{4}$ Faculty of Computer Science and Information Technology, Universiti Malaysia Sarawak (UNIMAS), 94300 Kota \\ Samarahan, Sarawak, Malaysia. \\ ${ }^{5}$ Centre for Computed Tomography and Industrial Imaging (CCTII), Malaysian Nuclear Agency, \\ Bangi, 43000 Kajang, Malaysia
}

\begin{tabular}{l}
\hline Article Info \\
\hline Article history: \\
Received Jun $12^{\text {th }}, 2015$ \\
Revised Aug $20^{\text {th }}, 2015$ \\
Accepted Aug $26^{\text {th }}, 2015$ \\
\hline
\end{tabular}

\section{Keyword:}

Evolutionary

Fan-beam

Prototype

Radon Transform

GUIDE

Sinogram

CT Scanner

\begin{abstract}
The development of software application for image reconstruction system is essential in the research study of computed tomography (CT). An experimental prototype was the research tool used in developing a series of useful prototype to evaluate the reconstruction methods. A CT scanner software prototype was created using the MATLAB image processing toolbox together with the Graphical User Interface (GUI) and Graphical User Interface Development Environment (GUIDE). The prototype model was selected to guide the development of the prototype application and mainly to provide a platform to update a system from the available knowledge. The prototype model was capable in creating a CT scanner synthetic datasets, display a sinogram image from the synthetic datasets, construct images using back projection technique and displaying cross-section images. Two types of images were acquired from this research, the first type was the unfiltered and blurred image and the second type was the filtered and clear image. This research also focused on two-dimensional parallel-beam and fan-beam geometry configuration; the principle of transforming fan-beam datasets into parallel-beam datasets was carried out using the classical rebinning concept
\end{abstract}

Copyright $(2016$ Institute of Advanced Engineering and Science. All rights reserved.

\section{Corresponding Author:}

M.S.M. Yusoff, R. Sulaiman,

Institute of Visual Informatics,

National University of Malaysia (UKM),

43600 Bangi, Selangor, Malaysia.

Email: soyapi81@gmail.com; riza@ivi.ukm.my

\section{INTRODUCTION}

The prototyping model approach has much been practised in the development of computerized application systems such as for management information system and decision support system. This study implements a prototype model approach to develop an application for image reconstruction of computerized tomography (CT). The prototype model approach consists of four steps of interactive processes between system developers and users of the system [1]. Figure 1 depicts an illustration of the four-step interactive processes for the prototype model approach to which has been adapted in the development of a CT image reconstruction application. Initial versions of a prototype are basically defined and constructed by system developers and later used by consumers mainly to identify any flaws within the system. In the case that a problem is found during the system implementation process, system developers are required to take 
appropriate actions to improve the existing prototype of the image reconstruction applications. A prototype model approach can be divided into three different types according to their respective functions that are known as throw-it-away prototyping, evolutionary prototyping and incremental prototyping [2]. For example, the evolutionary prototyping approach develops a system progressively where this method has a functional version during the prototype system development. Therefore, feedbacks from the user can be obtained either from the current phase or the phase of prototype development through to the final product [3].

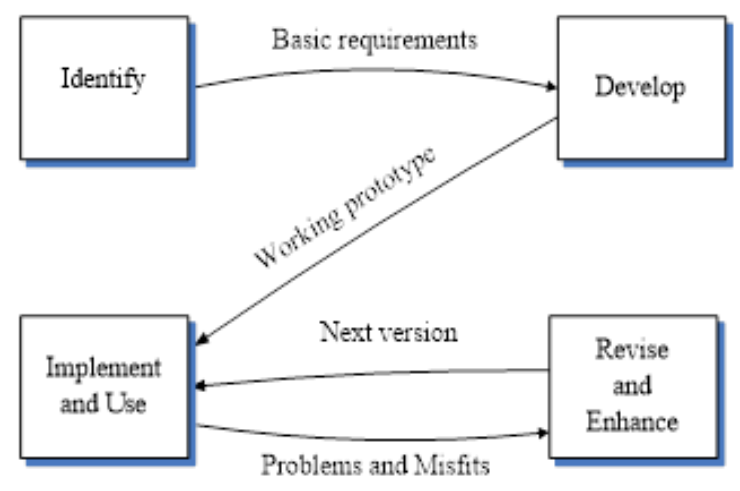

Figure 1. The Prototype Model

The main benefit of using a prototype model approach in comparison to the traditional model of system development life cycle (SDLC) is that a written documentation of the design specifications needs not to be provided by system developers. In addition, the prototype model approach has a short development period when developing an initial prototype for the user. Moreover, the development of a computerized system using the prototype model approach helps system developers to obtain detailed information and requirements about the system from a user perspective as well as to provide a better understanding regarding the users' environment. Further, user interaction with the prototype system provides the users with access and permission to deliver their responses like comments and suggestions to the system developers. These valuable responses of the user allow system developers to incorporate any necessary changes or improvement to the prototype system during the next prototype life-cycle series [4]. In this study, an evolutionary research prototype model for two-dimensional image reconstruction was developed using MATLAB applications.

\section{RESEARCH METHOD}

Microwave penetration in a variety of specific materials had provided light for the development of a prototype application for image reconstruction aimed in obtaining information on the internal structure of a physical object [5]. A mathematical model of computed tomography (CT) image reconstruction is defined as a quantitative explanation of radiation interaction with the particle object matter. The ability of a certain object to absorb and reduce the radiation rate is measured as the attenuation coefficient $(\mu)$ in the Beer's law, where the mean of $\mu$ corresponds to a linear integral transformation called Radon transformation [6].

The MATLAB image processing toolbox has the internal functions of the Radon transform and inverse Radon transformation that could help accelerate the development of prototype CT image reconstruction. Synthetic data acquisition through Radon transformation by MATLAB application requires source images known as the phantom image. The phantom image is the image of an object used to analyze or assess the quality of CT images. The phantom image can be classified into physical phantom to measure the real object and virtual phantom for mathematic simulation [7].

The CT can be divided into several types according to actual usage such as X-ray transmission CT, emission tomography (ET), magnetic resonance tomography (MT) and a less significant usage from ultrasound to infrared application [8]. Generally, a medical imaging system is categorized by transmission data and emission data. A transmission data places radiation sources outside a patient's body, while an emission data injects the radiation sources (isotopes) that radiate photons into the patient's bloodstream. In ET, a gamma detector rotates around the subject to count the photons that are emitted from the patient with the objective to obtain images of the anatomy's function [9]. On the other hand, the transmission data tomography is suitable for obtaining the transparency of the anatomical structure. 


\subsection{CT Scanner Geometry}

The CT scanner is known as a diagnostic tool used to observe the internal properties of an object [10]. The basic principles and the evolution of CT scanner were explained in [11, 12]. A brief description of the evolution of CT scanner is shown in Table 1.

Table 1. The generations of CT scanner

\begin{tabular}{ll}
\hline CT Scanner Generation & CT Scanner Description \\
\hline First-generation & $\begin{array}{l}\text { The } 1^{\text {st }} \text { generation of CT scanner was presented in 1972. This generation used an arrangement of linear } \\
\text { translation and incremental rotation or merely translate-rotate scanner [11], [12]. } \\
\text { The } 2^{\text {nd }} \text { generation of CT scanner was intended to reduce the scan time taken by the previous } \\
\text { generation and was introduced in the late 1974. This generation used multiple narrow beams from a } \\
\text { single radiation source and multiple detectors. The scanner was called rotate-translate [11], [12]. } \\
\text { The } 3^{\text {rd }} \text { generation of CT scanner was proposed in the late } 1975 \text { and remained to obtaining a faster } \\
\text { scan as compared to the previous generation. The CT scanner detectors array was linked to the X-ray } \\
\text { Third-generation } \\
\text { tube and rotated together around the object. The arrangement was referred to as rotate-rotate } \\
\text { movement [11], [12]. } \\
\text { In 1976, the } 4^{\text {nd }} \text { generation of CT scanner generation was proposed. This generation integrated large } \\
\text { stationary ring of detectors and the X-ray tube alone rotating around the object. Therefore, this } \\
\text { generation is able to measure rays at any distance from the centre of rotation. Further, this generation } \\
\text { can be dynamically calibrated before it passes in the object's shadow [11]. } \\
\text { The } 5^{\text {th }} \text { CT scanner is referred to as a multi-slice CT scanner of a multiple-row detector array. This } \\
\text { generation used a large ring to circle an object [12]. }\end{array}$ \\
\hline
\end{tabular}

Figure 2(a) shows an illustration of a parallel-beam computed tomography (CT) scanning system that uses an array of equally spaced radiation sources and an array of detector elements in which the radiation energy travels through the object and is expressed by the function $\mu(\mathrm{x}, \mathrm{y})$ that represents the attenuation coefficient of the radiation sources traversed along a straight line [13]. Further, Figure 2(b) illustrates the concept of one-dimensional projection datasets of the line integrals $\mathrm{P}(\mathrm{r}, \theta)$, where $\mathrm{r}$ is the line distance that is measured from the origin of the coordinate and $\theta$ is the angle of rotation, where measurements are taken [14].

In reality, the transmission data CT of fan-beam geometry is greater than parallel-beam geometry because it is more proficient for acquiring multiple projections simultaneously at different angles. Moreover, the emission CT of the fan-beam geometry enlarges the image and escalates sensitivity of the detection [15].

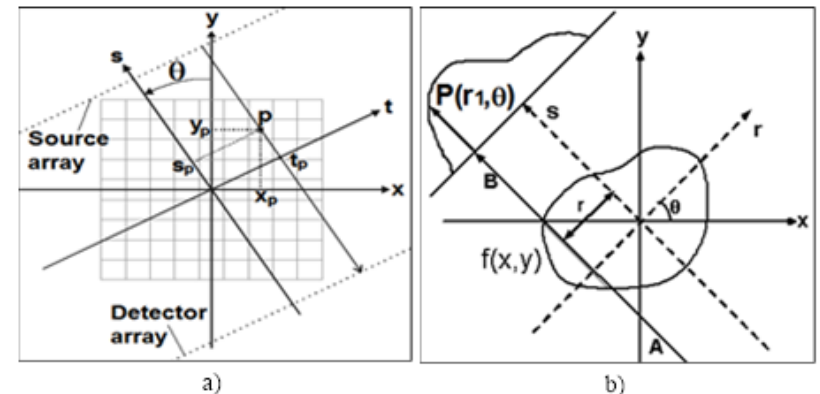

Figure 2. The parallel-beam geometry model, a) the source-detector array

b) The attenuation coefficient profile

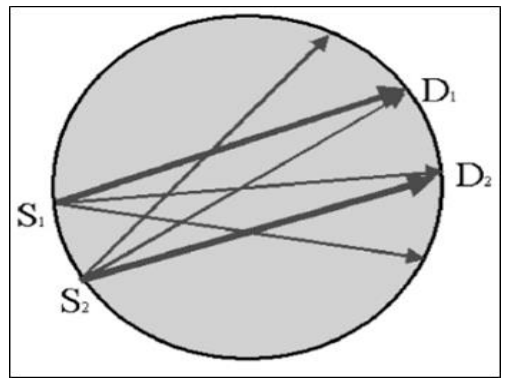

Figure 3. A concept of fan-beam rebinning framework 
The problems of image reconstruction in respects to fan-beam geometry are solved by rebinning fanbeam geometry into parallel-beam geometry [16]. Figure 3 shows an illustration of a conceptual fan-beam rebinning framework, where $\mathrm{S}$ represents a source of radiation and $\mathrm{D}$ represents a fan-beam detector. The parallel-beam dataset from the rebinning method can be reconstructed into CT image using inverse Radon transformation.

\subsection{Radon Transform}

The Radon transform is the transformation of two-dimensional Cartesian coordinate system of a graph function $\mathrm{f}(\mathrm{x}, \mathrm{y})$ into a two-dimensional Polar coordinate system of a distance and an angle represented by the function $g(\rho, \theta)$ [17]. This study used synthetic datasets of Radon transform to represent the datasets from the data acquisition system. The synthetic datasets are the image pixels that go through the Radon transformation. In other words, the pixels in Cartesian space of the images are transformed into the Radon space. The Radon transform of a linear integral for the function $\mathrm{P}(\theta, \mathrm{t})$ satisfies an area of $0 \leq \theta<2 \pi,-\infty<$ $t<\infty$, where $\theta$ represents an angle of rotation and $t$ represents a distance from the center of rotation [18]. The Radon transform can be used to turn the intensity pixels of phantom images into synthetic datasets for the computed tomography (CT) scan. The datasets from the CT scan machine is called sinogram. Thus, a sinogram can also be referred to as the Radon transform datasets.

Equation 1 shows the Radon transformation function, where the integral function of $f(x, y)$ represents the cross-sectional of the object to be reconstructed and the symbol $\delta$ is the Dirac delta function of the trigonometry that represents the rotation of the CT scanner. In the meantime, the function $\mathrm{g}(\mathrm{s}, \theta)$ represents the results of the liner integral function that measures the overall movement. The double integration of twodimensional Radon transformation is considered vital for CT scanner image reconstruction research as the rearrangement of projections at various angles and directions could exemplify the actual motion of the CT scanner radiation sources and detectors [19].

$$
g(s, \theta)=\iint_{-\infty}^{\infty} f(x, y) \delta(s-x \cos \theta-y \sin \theta) d x d y
$$

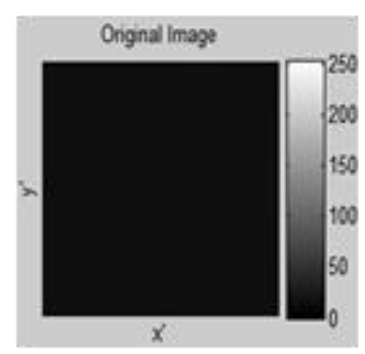

a)

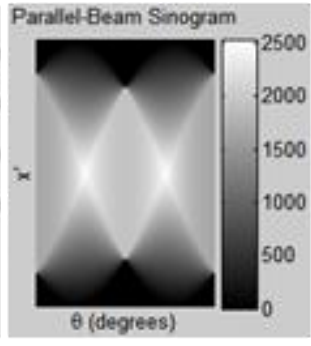

b)

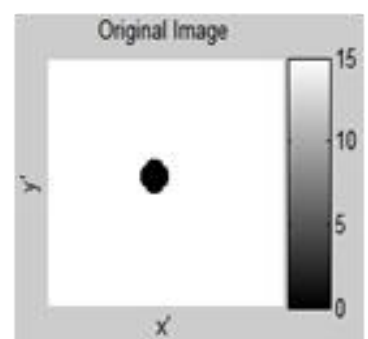

c)

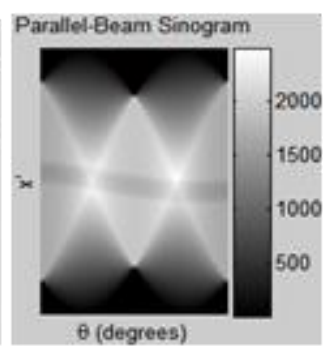

d)

Figure 4. The Sinograms of Parallel-Beam Projection

a) The blank image b) The sinogram of a blank image c) The dot image d) The sinogram of a dot image

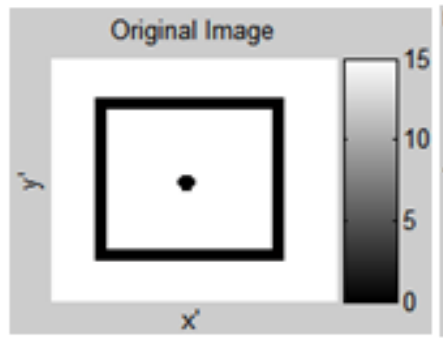

a)

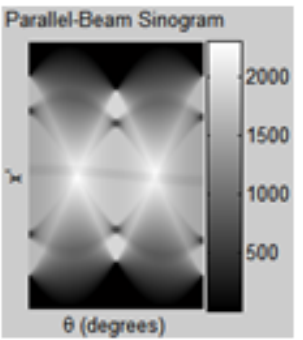

b)

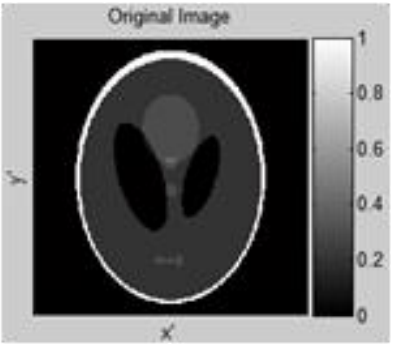

c)

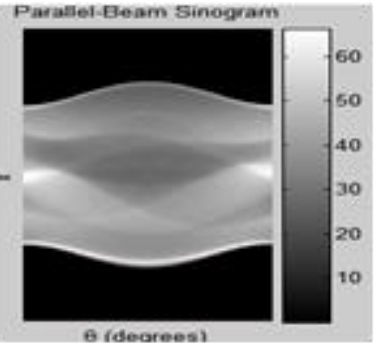

d)

Figure 5: The Sinograms of Parallel-Beam Projection

a) The dot and rectangle image b) The sinogram of dot and rectangle image c) The Shepp-Logan image d) The sinogram of the Shepp-Logan image 
Figure 4(a) shows a blank rectangle space without any image for control study purposes, and Figure 4(b) shows the sinogram image of the blank rectangle in parallel-beam space. The synthetic datasets are the pixel intensity of the sinogram image in gray scale level from 0 to 255 . Figure 4(c) shows a dot image at the middle of the rectangle. Figure 4(d) shows the sinogram image of the dot in the parallel-beam space. It is shown obvious in the sinogram space, that the dot image is transformed into a long curved line.

Figure 5(a) shows a dot image and rectangle image. Figure 5(b) shows the sinogram image of a dot image and the rectangle image in parallel-beam space. Furthermore, Figure 5(c) shows the Shepp-Logan image, and Figure 5(d) shows the sinogram of the Shepp-Logan image in parallel-beam space

Figure 6(a) shows the original Shepp-Logan image, while Figure 6(b) shows the sinogram of the Shepp-Logan image in fan-beam space.

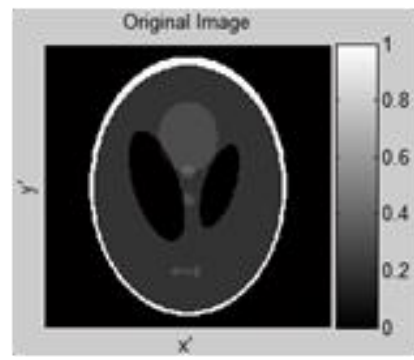

a)

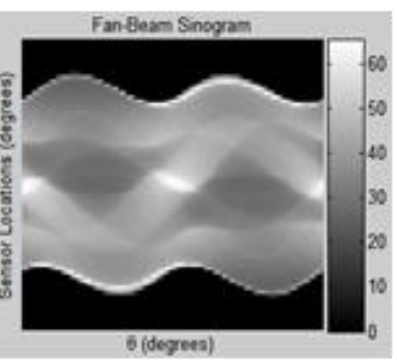

b)

Figure 6: The sinograms of fan-beam projection

a) The Shepp-Logan image b) The sinogram of the Shepp-Logan image

The program was written by Justin K. Romberg mainly to create synthetic datasets using MATLAB language, was viewed as an acceptable research tool to represent a physical data acquisition system in a virtual environment [20].

\section{RESULTS AND ANALYSIS}

The computer system platform for the development of the computed tomography (CT) prototype used Windows 7 operating system. The software application used was MATLAB version 2008a. The hardware system requirements used were Intel Core i5-2410M, CPU (2.3GHz), 4GB DDR3 RAM with 750 GB HDD.

\subsection{The Prototype Application}

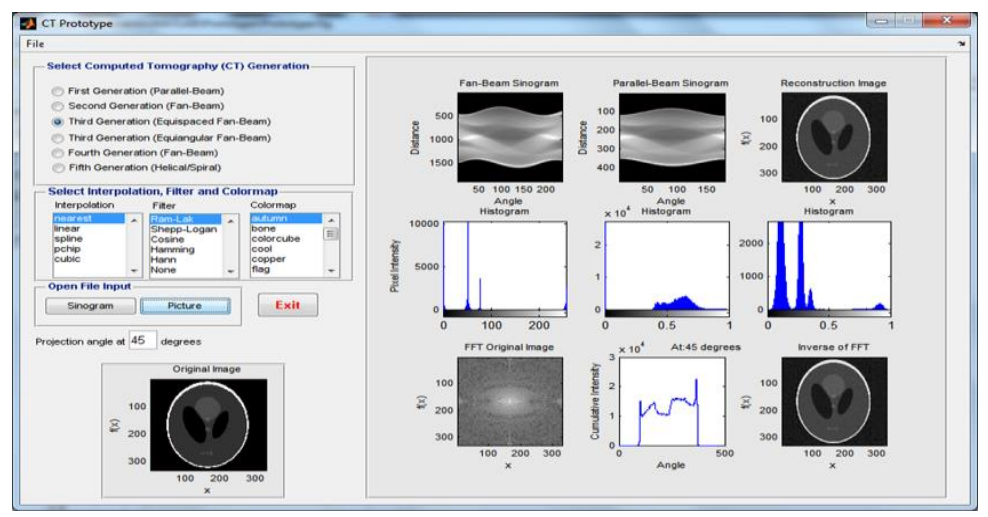

Figure 7. The CT Prototype for Image Reconstruction

Figure 7 shows the user interface of the computed tomography (CT) prototype application that was developed using MATLAB toolbox for the image reconstruction system. The user input was divided into four groups which are shown in Figure 8(a), 8(b), 8(c) and 8(d). Figure 8(a) shows the screen shoot for the 
selections of CT generation specifications as shown in Table 1. This selection option allows the user to experience with different generations of CT scanner evolutions. The selection option evaluated was the third generation equispaced fan-beam CT scanner. Figure 8(b) shows the original image used to generate synthetic datasets. Figure 8(c) shows a selection of five options for interpolation, 6 options for filter and 16 options for colour mapping. The user default values were provided from the nearest interpolation, Ram-Lak filter and autumn colormap scheme. Figure 8(d) shows the command buttons for the user to interact with the computer program and to start the reconstruction operation.

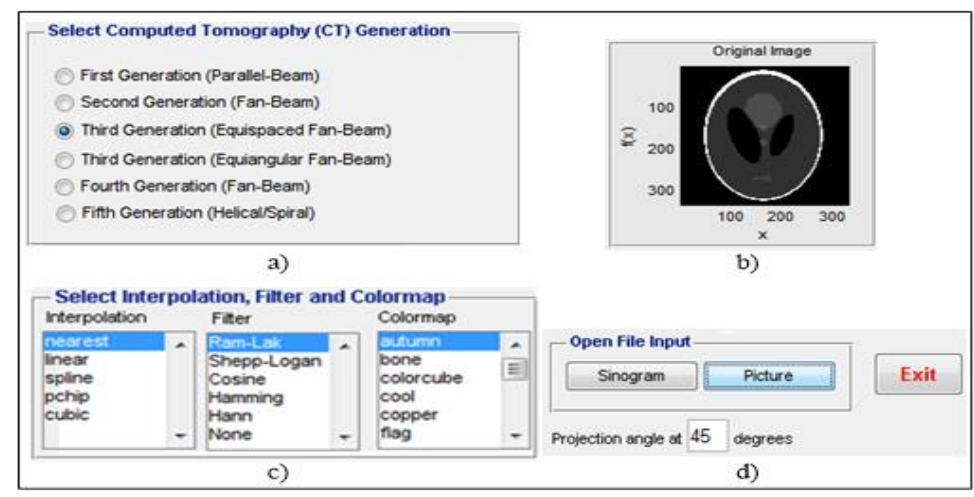

Figure 8. The User Input for the CT Prototype

a) The user selection for CT scanner generations b) The original image c) The user selection for interpolations, the filters and the color maps d) The command button and the input angle

The prototype provides the user with two buttons to open the input file. The first button labelled as "Picture" is a function to read a graphic file and the second button labelled as "Sinogram" is a function to read a numeric file. The projection angle text box is used to provide numeric data to draw a graph at a certain degree of CT scan rotation.

Figure 9 shows the output images from the CT prototype. The first row of images shows the fanbeam sinogram, the parallel-beam sinogram and the reconstruction image using filtered backprojection technique, respectively. The second row of images shows the distribution of data in the histogram for each of the first row CT scanner images. Lastly, the third row shows the one-dimensional Fast Fourier Transform (FFT) of the original image, the summation intensity of a projection of 45 degrees and the two-dimensional inverse Fast Fourier Transform (IFFT) image of the FFT function, respectively. The FFT function is a onedimensional complex number of linear transformation from the time domain to the frequency domain [21] where the output given is similar to the sinogram or synthetic datasets.

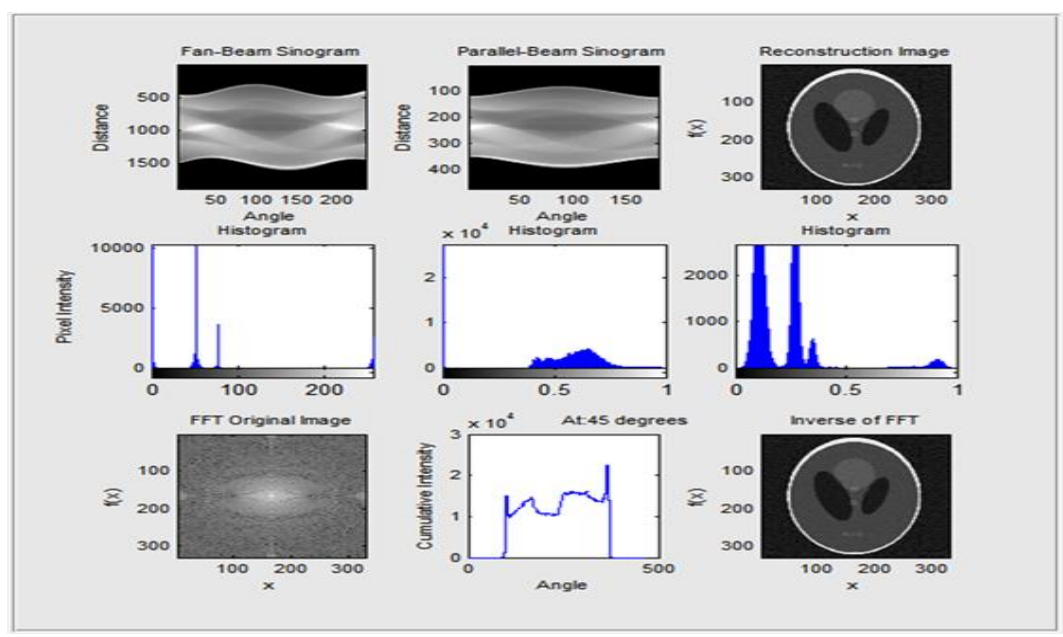

Figure 9. The User Output for the CT Prototype 


\section{CONCLUSION}

The computed tomography (CT) prototype application was developed using MATLAB program language to study the image reconstruction of CT by focusing on the construction of divergent beam and parallel beam in two-dimensional space. The prototype model of the system development life cycle (SDLC) was used to assist the development of the prototype image reconstruction application. In addition, the MATLAB application has many built-in functions, which are helpful in areas of image processing. Therefore, CT prototype was implemented using MATLAB image processing. The CT prototype application was developed based on the research methods of analytical approach for parallel beam and divergent beam CT system. Further study is recommended to lengthen the research on statistical image reconstruction methods and three-dimensional geometry space for cone beam image reconstruction.

\section{ACKNOWLEDGEMENTS}

This research was supported by the National University of Malaysia research grant HEJIM-FTSMFKAB-MTDC-101101005. The authors would like to acknowledge special thanks to Mohd Ikram Mohammad from the Rubber Research Institute of Malaysia (RRIM) and other colleagues rendering their technical support and assistance.

\section{REFERENCES}

[1] J. D. Naumann and A. M. Jenkins. Prototyping: The New Paradigm for Systems Development. MIS Quarterly. 1982; 6(3): 29-44.

[2] C. Floyd. A Systematic Look at Prototyping, in A Systematic Look at Prototyping. In Approaches to Prototyping: Springer-Verlag. 1984; 1-18.

[3] S. Hekmatpour. Experience with evolutionary prototyping in a large software project ACM SIGSOFT software engineering notes. 1987; 12(1): 38-41.

[4] M. A. Janson and L. D. Smith. Prototyping for Systems Development: A Critical Appraisal. MIS Quarterly. 1985; 9(4): 305-316.

[5] B. Yazgan, S. Paker, and M. Kartal. Image reconstruction with diffraction tomography using different inverse Radon transform algorithms. Biomedical Engineering Days, 1992., Proceedings of the 1992 International. 1992; 170-173.

[6] C. L Epstein. A Basic Model for Tomography, in Society for Industrial and Applied Mathematics, 2007.

[7] Z. Zakaria, N. H. Jaafar, N. A. M. Yazid, M. S. B. Mansor, M. H. F. Rahiman, and R. A. Rahim. Sinogram concept approach in image reconstruction algorithm of a Computed Tomography System using MATLAB. Computer Applications and Industrial Electronics (ICCAIE), 2010 International Conference. 2010; 500-505.

[8] L. Shepp. From Convolution Algorithms To Maximum Likelihood. Nuclear Science Symposium, 1990. Conference record : Including Sessions on Nuclear Power Systems and Medical Imaging Conference, 1990 IEEE. 1990; 1441 1444.

[9] K. Chidlowy and T. Möller. Rapid Emission Tomography Reconstruction, Simon Fraser University, Burnaby, B.C., Canada. 2003; 15-25.

[10] Y. Liu, Q. Yanshan University, X. Wang, Q. Yanshan University, M. Takei, and U. Nihon. SolidLiquid TwoPhase Flow Image Reconstruction Based on ERT Technique in Microchannel. TELKOMNIKA Indonesian Journal of Electrical Engineering. 2013; 11(1): 173-180.

[11] L. W. Goldman. Principles of CT and CT Technology. Journal of Nuclear Medicine Technology. 2007; 35(3): 115128.

[12] H. Young, J. Seung-Oh, and P. Jung-Byung. Fast image reconstruction from fan beam projections using parallel digital signal processors and special purpose processors. TENCON 99. Proceedings of the IEEE Region 10 Conference. 1999; 1558-1561.

[13] S. Coric, M. Leeser, E. Miller, and M. Trepanier. Parallel-beam backprojection: An FPGA implementation optimized for medical imaging. Monterey, CA. 2002; 217-226.

[14] A. C. Kak and M. Slaney. Principles of computerized tomographic imaging. IEEE Press, New York, NY. 1988.

[15] G. L. Zeng. Nonuniform noise propagation by using the ramp filter in fan-beam computed tomography. Medical Imaging, IEEE Transactions. 2004; 23(6): 690-695.

[16] G.-H. Chen. A new framework of image reconstruction from fan beam projections. Medical Physics. 2003; 30(6): 1151-1161.

[17] C. Høilund. The Radon Transform: Aalborg University, 2007.

[18] W. R. Madych. Radon's Inversion Formulas. Transactions of the American Mathematical Society. 2004; 356(11): 4475-4491.

[19] S. G. Azevedo, D. J. Schneberk, J. P. Fitch, and H. E. Martz. Calculation of the rotational centers in computed tomography sinograms. Nuclear Science, IEEE Transactions. 1990; 37(4): 1525-1540.

[20] C. Pan. Image Processing Using SPECT Analysis by The Five Fearless Thinkers. Rice University; 1996 [08/11/2012]; Available from: http://www.clear.rice.edu/elec431/projects96/DSP/projections.html. 
[21] X. Xu and U. China Three Gorges. FFT Analysis on Coupling Effect of Axial and Torsional Vibrations in Circular Cross Section Beam of Steam Turbine Generators. TELKOMNIKA Indonesian Journal of Electrical Engineering. 2014; 12(2): 1625-1633.

\section{BIOGRAPHIES OF AUTHORS}
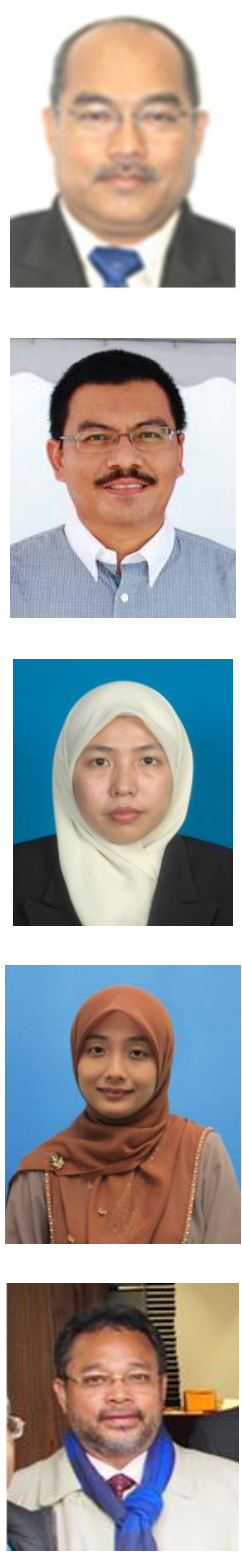

Mohd Soyapi Mohd Yusoff studied at the National University of Malaysia (Universiti Kebangsaan Malaysia, UKM) where he received a bachelor degree (Hons.) in Information Technology in 2008. Currently, he is pursuing a MSc in Information Technology at the Institute of Visual Informatics, UKM. He is profound in conducting researches on the subject of image reconstruction of computed tomography. His research interests in the area of computer science and technology also includes statistic, optimization, neural network, signal processing, parallel computing, image reconstruction/filtering and image processing.

Riza Sulaiman is an Associate Professor and a Senior Research Fellow in the Institute of Visual Informatics, National University of Malaysia (Universiti Kebangsaan Malaysia, UKM). He holds a PhD in Mechanical Engineering, a MSc in Advanced Manufacturing Technology from the University of Portsmouth and B.Eng. (Hons) in Mechanical Engineering from the University of Sunderland, United Kingdom. His research area is in CADCAM and Visualisation. He is a member of the Institution of Mechanical Engineers (IMechE), United Kingdom and the Board of Engineers Malaysia (BEM).

Kamarudin Shafinah received a MSc degree (Management Information System) in 2009 from Universiti Putra Malaysia (UPM). She joined the Faculty of Agriculture and Food Sciences, Universiti Putra Malaysia Bintulu Campus, Sarawak, Malaysia as tutor since 2005. Her research interests include information system and management in agriculture domain, user acceptance towards new technology, requirement engineering as well as human computer interaction. Currently, she is pursuing her PhD in Universiti Kebangsaan Malaysia (UKM).

Ramli Fatihah holds a B.IT. in Management Information Systems (2002) from Universiti Malaysia Sabah (UMS) and M.IT (2004) from Universiti Malaya (UM). She joined the Faculty of Computer Science and Information Systems, Universiti Malaysia Sarawak (UNIMAS) as a lecturer since 2004. Currently, she is pursuing her Ph.D at Universiti Kebangsaan Malaysia (UKM). Her current research interests include Semantic Technology and Information Retrieval.

Jaafar Abdullah is Manager and Principal Research Scientist of Plant Assessment Technology Group, Industrial Technology Division, Malaysian Nuclear Agency, Malaysia. He has been involved in a wide variety of research and development using radioisotope (sealed source and radiotracer) technology for industrial plant assessment and non-destructive evaluation since 1980. As a pioneer of radioisotope technology in Malaysia and the region, his experience in developing the technology for investigating various types of process equipment in petroleum refineries, chemical plants, gas processing installations and power generation utilities is extensive. He is recognised by the International Atomic Energy Agency (IAEA) as an expert and consultant in the field of industrial applications of radioisotope technology. He received his BSc (Physics), MSc (Nuclear Technology) from University of Surrey, UK and PhD (Materials Engineering) from University of Wales Swansea, UK. He has published substantial amount of scientific papers, consultant documents, technical reports, promotional articles and books. He has won a number of awards and recognitions both nationally and internationally for his outstanding innovations in the peaceful use of nuclear technology in industry and engineering. $\mathrm{He}$ is presently the president of Malaysian Society for Computed Tomography and Imaging Technology (MyCT). He was the chairman of Malaysian Computed Tomography Interest Group (CTiG) and was the chairman of technical \& training committee of the Malaysian Society for Non-destructive Testing (MSNT). He is also a member of other professional organisations and societies, which include MNS, IPM, IMM, MARS and MARPA in Malaysia 